Photogallery

\title{
Live autofluorescence in planulae of Acropora tenuis
}

\section{Eiji FUJIWARA ${ }^{1}$, Kanae MATSUSHIMA ${ }^{2}$, and Masayuki HATTA ${ }^{2, *}$}

${ }^{1}$ Documentary Channel Co. Ltd., Tsurugaoka 851-1, Tsurugashima, Saitama 350-2204, Japan

${ }^{2}$ Graduate School of Humanities and Sciences, Ochanomizu University, Tokyo 112-8610, Japan

* Corresponding author: M. Hatta

E-mail: hatta.masayuki@ocha.ac.jp

Communicated by Michio Hidaka (Editorial Board Member)

Keywords Acropora, Coral, Fluorescence, Neuron, Planula

Planula larvae of acroporids reveal strong autofluorescence all over the body after treatment with aldehyde fixatives (Fig. 1A), but live planulae of Acropora tenuis show different autofluorescence. Green fluorescence by B-excitation revealed typical bipolar neurons with varicosities along axons (Fig. 1B), even though these neurons were only seen in small numbers of planulae, and only sparsely. In contrast, red fluorescence by G-excitation revealed numerous rod-like structures dotted in the ectodermal layer over the whole body (Fig. 1C-E) of all planulae so far observed (more than 30 individuals). These signals resemble the cell body of neurons visualized by immunohistochemistry targeting a neuropeptide
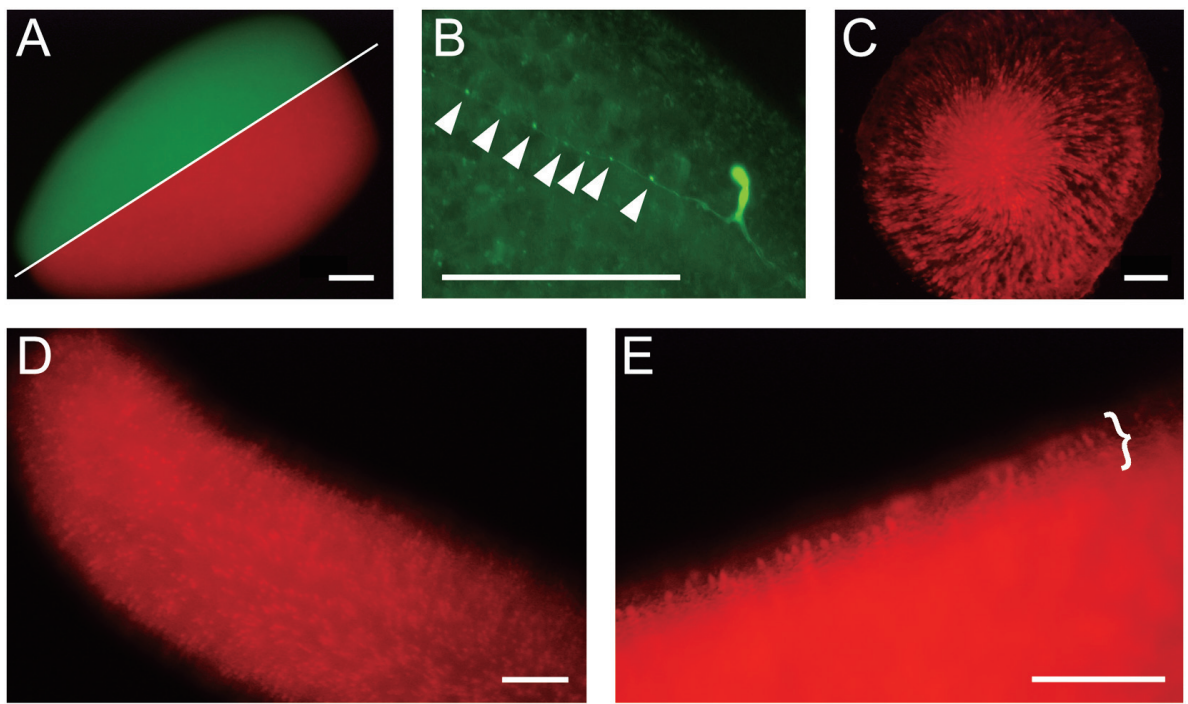

Fig. 1 Autofluorescence in A. tenuis planulae. A Fluorescence of a formalin-fixed planula. Upper left: green fluorescence by B-excitation. Lower right: red fluorescence by G-excitation. B A typical neuron detected by Bexcitation in a live planula. Arrowheads indicate varicosities. C-E Red fluorescence by G-excitation in live planulae. C View from the aboral side. D A side view. E A side view in higher magnification. The ectodermal layer is indicated by a bracket. (Scale bars $=100 \mu \mathrm{m}$ ) 
(Hayward et al. 2001), although direct evidence is missing to identify the cell type of these red fluorescent signals. Roles of the fluorescence still remain in the dark since fluorescent signals are different in other species and also polymorphic (Kenkel et al. 2011). This report first detected clear neuron structures in planula of Acropora, and may shed light on the importance of live imaging of fluorescence in corals.

\section{Acknowledgments}

We thank Akajima Marine Science Laboratory for sampling supports. This work was supported by Sasakawa Scientific Research Grant from the Japan Science Society to KM, and the Grant-in-Aid for Scientific Research (C) from MEXT Japan to MH.

\section{References}

Hayward DC, Catmull J, Reece-Hoyes JS, Berghammer H, Dodd H, Hann S, Miller DJ, Ball EE (2001) Gene structure and larval expression of cnox-2Am from the coral Acropora millepora. Dev Gens Evol 211: 10-19

Kenkel CD, Traylor MR, Wiedenmann J, Salih A, Matz MV (2011) Fluorescence of coral larvae predicts their settlement response to crustose coralline algae and reflects stress. Proc R Soc B 278: 2691-2697

Received: 13 January 2011/Accepted: 7 September 2012

(C) Japanese Coral Reef Society 